Nota clínica

\title{
Transuretero-ureterostomía espontánea después de estenosis completa de un conducto ileal, con cuatro años de seguimiento conservador
}

\author{
Luis A. Fariña Pérez, Daniel Pesqueira Santiago \\ Servicio de Urología. Hospital POVISA. Vigo. Pontevedra.
}

\begin{abstract}
Resumen
Introducción: La estenosis del conducto ileal es una complicación infrecuente de esta derivación urinaria. En el caso que describimos pasó durante un tiempo inadvertida y llevó al paciente a una situación de riesgo. Después de implantar una nefrostomía bilateral, se encontró una transuretero-ureterostomia espontánea.

Caso Clínico: Un varón de 70 años con un conducto ileal realizado 15 años antes por tener tumor vesical, ingresó con signos de infección intraabdominal grave y oliguria. Los estudios radiológicos mostraron absceso intraabdominal, y se encontró estenosis casi completa del conducto ileal, que se trató conservadoramente mediante una nefrostomia bilateral. Después de ser dado de alta ocurrió la caída accidental de la nefrostomía derecha, recogiendo cantidades normales de orina por la única nefrostomía izquierda. Se comprobó la existencia de una transuretero-ureterostomía espontánea, con estenosis de la porción distal del conducto ileal. Desde entonces permanece con la nefrostomía, que se cambia periódicamente y en cuatro años de seguimiento no ha tenido deterioro renal significativo.

Comentario: El origen de esta estenosis se atribuye a los cambios inflamatorios o inmunológicos en la pared del segmento intestinal, inducidos por la presencia crónica de la orina. Nuestro caso es singular por el curioso resultado de una transuretero-ureterostomía in situ y por el largo seguimiento conservador que se hizo, en un paciente que mantiene una calidad de vida buena.
\end{abstract}

Palabras clave: Conducto ileal. Complicaciones.

Spontaneous transuretero-ureterostomy after complete stenosis of an ileal conduit, with four years of conservative follow-up

\section{Abstract}

Introduction: Stenosis of an ileal conduit is a rare complication of this urinary diversion. In the case here described, such a complication was neglected for some weeks and left the patient in a high risk situation. After implantation of a bilateral nephrostomy, a spontaneous transuretero-ureterostomy was found.

Clinical case: A 70-year-old man with an ileal conduit performed 15 years before because of bladder tumour, was admitted with signs of severe intraabdominal infection and oliguria. The image studies shown intraabdominal abscess, and an almost complete stenosis of the ileal conduit, that was conservatively treated with a bilateral percutaneous nephostomy. After discharged, he reported an accidental falling-out of the right nephrostomy, collecting normal diuresis from the only left nephrostomy. The spontaneous appearance of a transuretero-ureterostomy was demonstrated. The patient refused surgery and remained with a nephrostomy that is periodically changed, and after four years of follow-up he has neither significant kidney dysfunction nor other incidences.

Comment: The origin of this ileal conduit stenosis is related to the inflammatory or immunologic changes induced by the chronic presence of the urine on the wall of the intestinal segment. This case is singular because of the curious result of an in situ transuretero-ureterostomy, and because of the long conservative follow-up, without significant complications, in a patient that keeps a good quality of life.

Keywords: Ileal conduit. Complications.

$\mathrm{L}$ a estenosis completa o parcial del conducto ileal es una de las complicaciones más infrecuentes de esta clásica derivación urinaria, que en los últimos años se realiza menos, por la tendencia a construir derivaciones urinarias ortotópicas continentes siempre que es posible. Esta complicación es poco conocida y se han descrito pocos casos en la biblio- grafía, pero es importante, porque puede conllevar dificultades diagnósticas y de tratamiento, poniendo en peligro la vida del paciente por el compromiso renal bilateral o por el cuadro infeccioso que genera. En este paciente que presentamos, se dan estas circunstancias, pues la complicación pasó durante tiempo inadvertida en otro Centro y llevó al paciente 
a una situación de riesgo vital. Se encontró una estenosis completa de la porción distal del conducto ileal, con el curioso resultado de una transuretero-ureterostomia espontánea, que se trató de forma conservadora, sin complicaciones significativas después de un seguimiento de cuatro años.

\section{CASO CLÍNICO}

Un varón de 70 años con una derivación urinaria de conducto ileal realizada 15 años antes por tener tumor vesical (estadio clínico no conocido), ingresó en un Servicio de Cirugía General con signos de infección intraabdominal grave. El estudio mediante TAC mostró absceso intraabdominal, y fue tratado con antibióticos durante unos días, sin llegar a un diagnóstico definitivo. Después de ser trasladado a nuestro Hospital, con un cuadro de oligoanuria, insuficiencia renal y persistencia de signos de infección intraabdominal, con plastrón en la fosa ilíaca derecha, se encontró hidronefrosis bilateral, fuga urinosa en la vecindad del conducto ileal, responsable del cuadro infeccioso intraabdominal, y fístula urinaria desde el conducto ileal proximal a asas de ileon distal (Fig 1), así como estenosis casi completa del conducto ileal (Fig 2). Se trató conser- vadoramente mediante una nefrostomia bilateral, y fue dado de alta para continuar el tratamiento antibiótico en casa. Unos días después, en la siguiente consulta, refiere la caída accidental de la nefrostomía derecha, recogiendo cantidades normales de orina por la única nefrostomía izquierda. Comprobamos la existencia de una transuretero-ureterostomia espontánea, con estenosis de la porción distal del conducto ileal (Fig 3A). Se le ofreció la posibilidad de tratamiento quirúrgico, con la opción de construir un nuevo conducto ileal o la reconversión a un conducto colónico transverso, lo que rechazó por encontrarse cómodo con la situación que tenía. Subsecuentemente, permanece con la nefrostomía (sonda de balón), que se cambia una vez al mes y el conducto ileal está sin función. En cuatro años de seguimiento no ha tenido deterioro renal funcional significativo, ni otras incidencias (Fig 3B).

\section{COMENTARIO}

El origen de la estenosis del conducto ileal no está aclarado, pero se ha atribuido un papel determinante a los cambios inflamatorios o inmunológicos inducidos por la presencia crónica de la orina y/o de gérmenes en el segmento intestinal, que

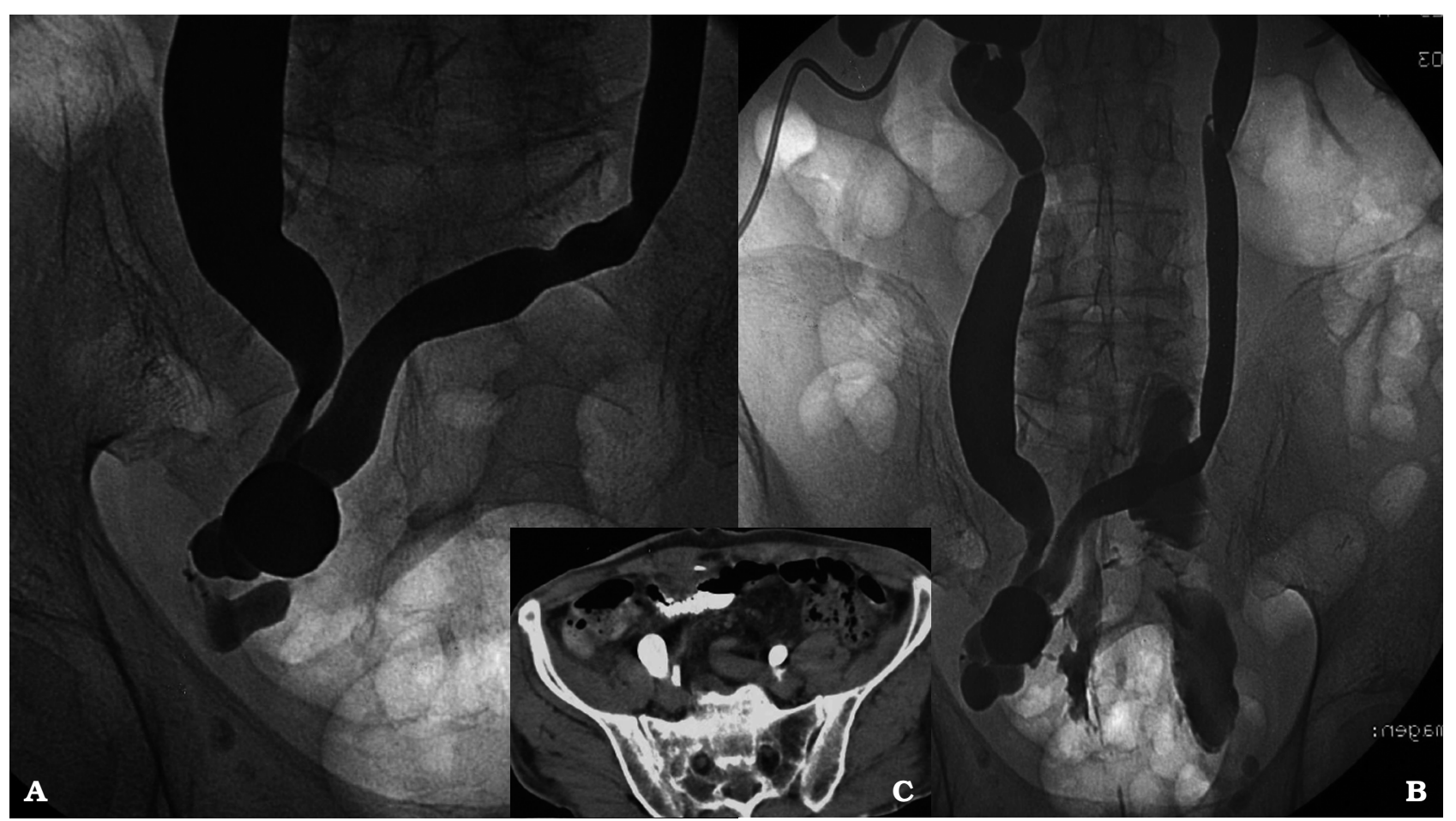

FIGURA 1. Pielografia bilateral por nefrostomia: se observa la estenosis completa del conducto en su porción proximal (A), con fuga intraabdominal del constraste (B), causante de infección intraabdominal y fistula que rellena asas de ileon distal (C, imagen de TAC) . 


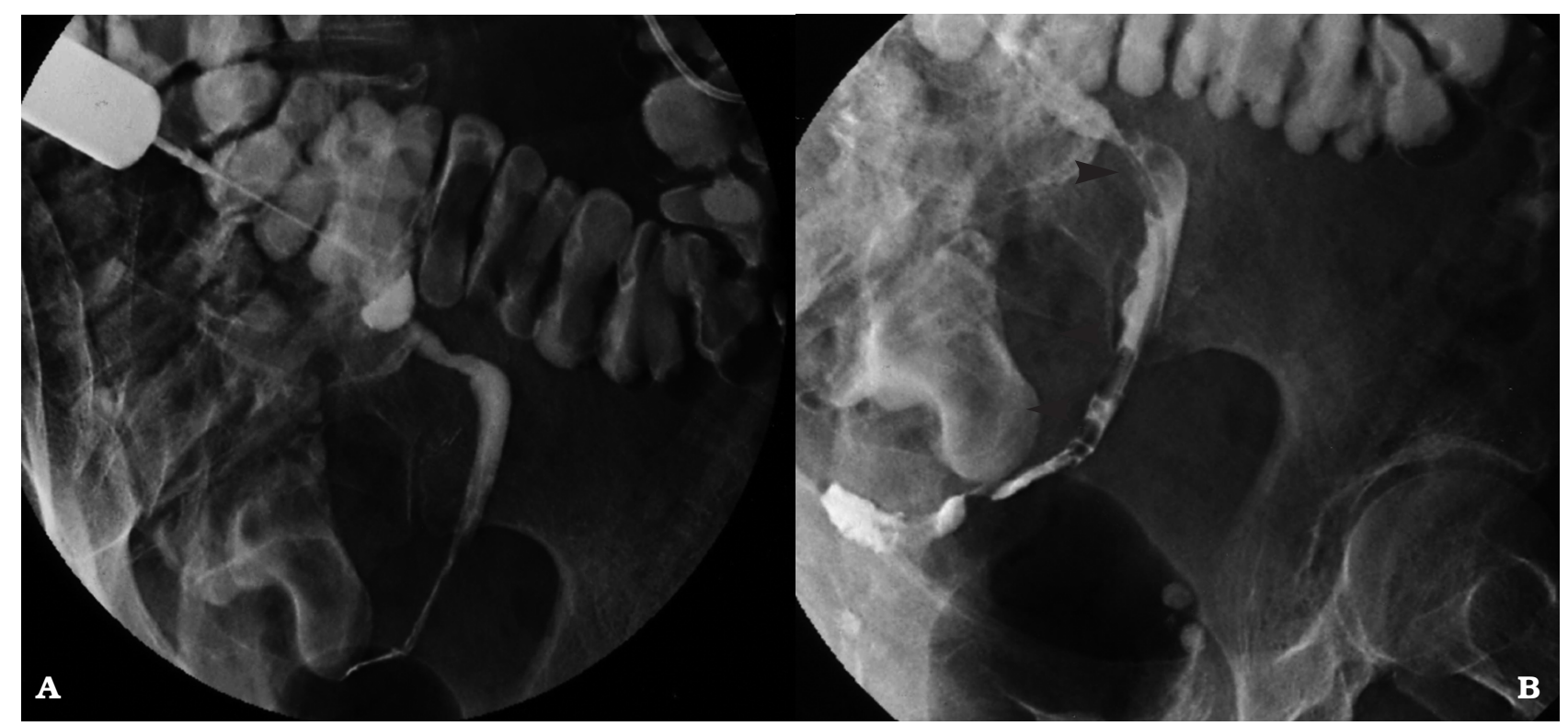

FIGURA 2. Conductografia: estenosis larga y arrosariada del conducto, sin encontrar reflujo ureteral. A la derecha el conducto ileal tiene una sonda y algunas burbujas de aire, hay contraste oral en colon.

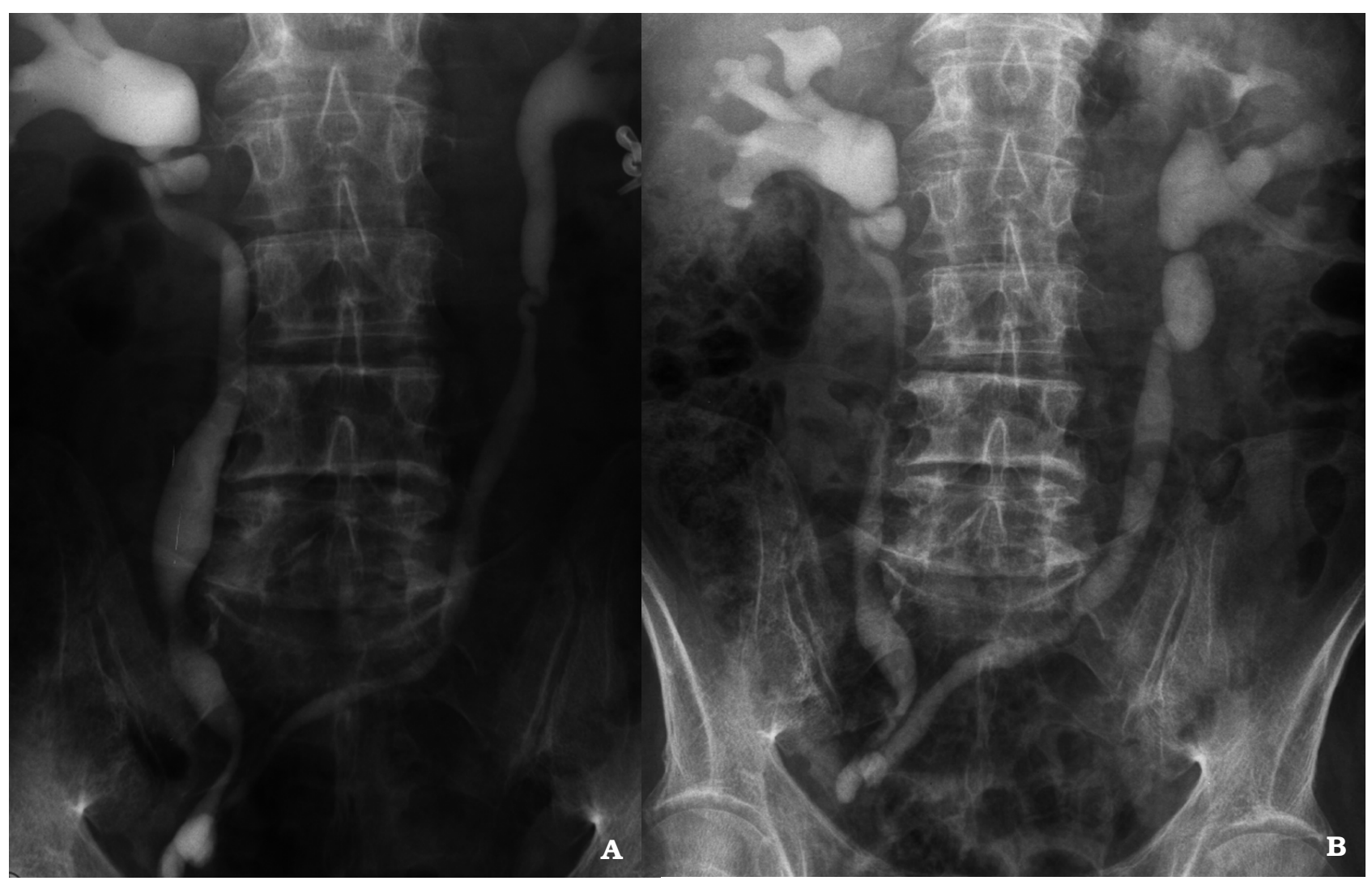

FIGURA 3. Pielografia anterógrada por la nefrostomia izquierda (A) y urografia endovenosa reciente (B) : se rellenan bien ambas vias urinarias, que tiene dilatación leve. A la derecha puede verse la sonda de nefrostomia con balón en cáliz superior de riñón izquierdo.

generarían un deterioro a largo plazo de la pared intestinal $^{1-3}$, con lesiones que predominan en la mucosa y submucosa, y son transmurales en los casos más graves. La isquemia intestinal segmenta- ria crónica por enfermedad vascular aterosclerósica concomitante no parece ser influyente, aunque el cuadro que aquí presentamos, de estenosis de instauración crónica, parecería similar al originado en 
una isquemia aguda por infarto trombótico o por volvulación del mesenterio ileal, que se presenta con crisis de anuria de forma aguda ${ }^{4}$. Una de estas formas de isquemia también parece ser el origen de las rupturas espontánea de las derivaciones ortópicas y ampliaciones vesicales, un cuadro temido por la peritonitis que le sigue. En el estudio histológico de estos segmentos intestinales perforados se ha visto, en efecto, la presencia de trombosis vascular e infarto isquémico mural, así como atrofia de la capa muscular de la pared intestinal ${ }^{5}$.

Aunque el cuadro clínico y radiológico de la estenosis del conducto ileal pueda ser típico, eventualmente será necesario hacer un diagnóstico diferencial, mediante los estudios radiológicos, endoscópicos y biópsicos apropiados, con la estenosis limitada al estoma, con otros cuadros de causa benigna que cursan con hidronefrosis, oliguria o anuria en el paciente con esta derivación urinaria ${ }^{6}$, y con complicaciones malignas, generalmente tardías, como la afectación por cáncer transicional de los uréteres, o neoplasia del segmento intestinal, más infrecuente .

El caso que describimos es singular por la sorprendente aparición de una transuretero-ureterostomía espontánea "in situ", que siguió a la estenosis completa de la porción distal del conducto ileal, hallazgo que para nuestro conocimiento no se ha- bía registrado previamente. El tratamiento conservador realizado, obviando otras opciones con mucha morbilidad, y el largo seguimiento que se hizo, sin encontrar complicaciones significativas, en un paciente que mantiene una calidad de vida buena, son también destacables.

\section{REFERENCIAS}

1. Mitchell ME, Yoder IC, Pfister RC, Daly J, Althausen A. Ileal loop stenosis: a late complication of urinary diversion. J Urol. 1977; 118(6):957-961

2. Guate JL, Velasco J, Lanzas JM, Tuero JG, Castellanos L, Naranjo FG. Estenosis de asa ileal en paciente con derivación urinaria tipo Bricker . Actas Urol Esp. 1997;21(10):981-984

3. Magnusson B, Carlen B, Bak-Jensen E, Willen R, Mansson W. Ileal conduit stenosis-an enigma. Scand J Urol Nephrol. 1996; 30:193197.

4. Coxon JP, Jones CR. Two cases of patients with renal transplants presenting with anuria due to an infarcted ileal conduit. Transpl Int. 2002;15(9-10):515-516.

5. Crane JM, Scherz HS, Billman GF, Kaplan GW. Ischemic necrosis: a hypothesis to explain the pathogenesis of spontaneously ruptured enterocystoplasty. J Urol. 1991;146(1):141-144.

6. Hétet JF, Rigaud J, Karam G et al. Complications des urétérostomies cutanées trans-iléales selon Bricker. Analyse d'une série de 246 patients. Prog Urol. 2005;15:23-29.

Correspondencia autor: Dr. Luis A. Fariña

Servicio de Urologia. Hospital POVISA

Salamanca, 5 - 36211 Vigo (Pontevedra). Tel.: 986413144

E mail: luisfarina@yahoo.com

Información artículo: Nota clínica

Trabajo recibido: abril 2007

Trabajo aceptado: mayo 2007 